\title{
Über eine spektroskopische Methode zum Nachweis geringster Mengen Wasser
}

\author{
Von Eva Sauter \\ Aus dem Kaiser-Wilhelm-Institut für Physik, Max-Planck-Institut, Hechingen \\ (Z. Naturforschg. 3 a, 392-395 [1948]; eingegangen am 1. April 1948)
}

\begin{abstract}
Es wird eine spektroskopische Methode angegeben, mit Hilfe des bei $\mathrm{H}_{2} \mathrm{O}$-Anregung auftretenden Zerfallsspektrums der OH-Gruppe Wasserbeimengungen bis zu 1:180000 in einer Glimmentladung nachzuweisen.
\end{abstract}

$\mathrm{D}$ er Versuch amerikanischer Forscher ${ }^{\mathbf{1}}$, mit Hilfe der Emissionsspektren eine Analyse von Gemischen organischer Substanzen durchzuführen, ließ erkennen, daß dies nicht möglich ist. Später beschritten H. S ch ül e r und A. Wo el$\mathrm{dike}^{2}$ einen neuen Weg zur Erzeugung von Emissionsspektren organischer Substanzen mit einer eigens dafür entwickelten Entladungsröhre, um damit die Vorgänge in komplizierteren Molekülen zu studieren. Auch bei der Durchführung dieser Versuche ${ }^{3}$ stellte sich heraus, daß eine allgemeine Spektralanalyse organischer Substanzen praktisch undurchführbar ist. Trotzdem ist es möglich, spezielle Fragestellungen dieser Art in experimentell günstigen Fällen $\mathrm{zu}$ bearbeiten.

Eines dieser Probleme, das auch von technischem Interesse sein dürfte, ist der Nachweis geringster Mengen Wasser àls Beimengung anderer, etwa organischer, Substanzen. Es galt vor allem festzustellen, bis $\mathrm{zu}$ welchen Empfindlichkeitsgrenzen der Nachweis getrieben werden kann.

Verfährt man analog der Spektralanalyse von Metallatomen, so liegt es nahe, das Auftreten der arteigenen Spektren der Moleküle für den Nachweis zu verwenden. Dabei gibt es verschiedene Möglichkeiten. Beim Wasser liegen die Verhältnisse folgendermaßen:

Bei Absorptions-Aufnahmen liegt das Elektronenbandenspektrum des $\mathrm{H}_{2} \mathrm{O}$ im Schumann-Gebiet $^{4}$, d. h. im Gebiet unterhalb $\lambda=1850 \AA$, das nur noch der Vakuumspektroskopie zugänglich ist. Das Arbeiten in diesem Gebiet ist experimentell schwierig, außerdem sind Platten und Spektrographen für Spurennachweise zu unempfindlich.

1 A ustin u. B l a c k, Physic. Rev. 35, 452 [1930].

2 H. Schüler u. A. Wo el d ike, Physik. Z. 42, 390 [1941].

${ }^{3}$ H. S c hül e r u. A. Wo eld i ke, Physik. Z. 43, 17 [1942] und folgende Arbeiten.
Unempfindlichkeit bei geringen Substanzmengen machen überhaupt die Absorptionsspektren für die vorliegenden $Z$ wecke ungeeignet. Der zweite noch mögliche Weg, die Feststellung des Auftretens des Rotations-Schwingungsspektrums, scheidet aus, da dieses Spektrum, wie bei allen anderen Molekülen, im Ultrarot liegt. Eine Trennung und genaue Zuordnung der einzelnen Banden ist dahèr bei einem Spurennachweis schwer möglich, zumal die Rotationschwingungs-Spektren nicht mehr photographisch erfaßbar sind, sondern nur mit Bolometern oder anderen Instrumenten zur Bestimmung der Wärmestrahlung abgetastet werden können.

Bei der Prüfung der $\mathrm{H}_{2} \mathrm{O}$-Banden in der Emission zeigt sich, daß alle Banden, die den Übergängen auf den Grundzustand entsprechen, im Schumann-Gebiet liegen, was, wie oben schon gesagt, ihre Verwendung hier unmöglich macht. Das an sich in Frage kommende Emissionsspektrum im Sichtbaren entspricht einem Übergang zwischen höheren Anregungszuständen ${ }^{5}$. Da bei der Anregung in der positiven Säule, wie sie hier vorliegt, die Besetzung der Elektronenzustände mit zunehmendem Abstand vom Grundzustand stark abnimmt, würde die Intensität dieses Spektrums im Sichtbaren für einen Empfindlichkeitsnachweis nicht ausreichen.

Es gibt nun in der Molekülspektroskopie eine neue Möglichkeit des Nachweises von Mischungskomponenten. Bei näherer Untersuchung der mit der neuen Entladungsröhre gefundenen Molekülspektren stellte sich nämlich heraus, daß neben den arteigenen Spektren der Verbindungen bei

${ }^{4}$ H. Sp on er, Molekülspektren I, Tabellen. Verlag J. Springer, Berlin 1935.

${ }_{5}$ H. Schüler u. A. Wo eld ike, Physik. Z. 44, 335 [1943]. 
zahlreichen Substanzen charakteristische Zerfallsspektren existieren. Der Prozeß dabei ist folgender: Das Molekül wird angeregt und zerfällt dann im angeregten Zustand ohne Einwirkung von Nachbarmolekülen selbständig in mindestens zwei Bruchstücke. Wenn nun beim Zerfall eines davon angeregt zurückbleibt, erscheint sein Spektrum und macht den Prozeß nachweisbar. Dieser Vorgang wird als „natürlicher Zerfall“ bezeichnet. Das Auftreten des Spektrums eines solchen Zerfallsproduktes ist also ein sicheres Anzeichen für das Vorhandensein des ursprünglichen Ausgangsmoleküls.

$\mathrm{Ob}$ es sich um die Produkte aus natürlichen Zerfallsprozessen handelt, entscheidet man experimentell in der Weise, daß man die Stromstärke, die durch die Entladung geht, etwa von $30 \mathrm{~mA}$ bis $3 \mathrm{~mA}$ variiert. Dabei zeigt sich, daß alle „sekundären“ Prozesse mit abnehmender Stromstärke an Intensität verlieren und praktisch verschwinden. Das Spektrum des Bruchstückes aus dem natürlichen Zerfall behält dagegen seine Intensität bei. Es ändert sich also das Intensitätsverhältnis zwischen den Zerfallsspektren zugunsten des Bruchstücks aus dem natürlichen Zerfall.

Beim Wasser, dessen Nachweis in den folgenden Experimenten ja geführt werden soll, ergeben sich nun bei der Anregung in der Gasentladung verschiedene Zerfallsmöglichkeiten, deren wichtigste hier angeführt seien:

$$
\begin{array}{ll} 
& \mathrm{OH}^{*}+\mathrm{H}^{*} \\
\mathrm{H}_{2} \mathrm{O}^{*} \rightarrow & \mathrm{OH}+\mathrm{H}^{*} \\
& \mathrm{O}+\mathrm{H}^{*}{ }^{*}+\mathrm{H}^{2}+\mathrm{H} \\
& \mathrm{OH} \mathrm{H}^{*}+\mathrm{H}^{*} \\
& \mathrm{OH}+\mathrm{H}
\end{array}
$$

\begin{tabular}{|c|c|c|}
\hline Angeregtes Bruchstück & Prozeß & Energien \\
\hline $\mathrm{OH}$ & $\mathrm{H}_{2} \mathrm{O}^{*} \rightarrow \mathrm{OH}^{*}+\mathrm{H}$ & $\left.\begin{array}{c}\mathrm{OH}_{\mathrm{OH}^{*}} \rightarrow \mathrm{H}=5,1 \mathrm{~V} \\
=4\end{array}\right\}=9,1 \mathrm{~V}$ \\
\hline $\mathrm{H}$ & $\mathrm{H}_{2} \mathrm{O}^{*} \rightarrow \mathrm{OH}+\mathrm{H}^{*}$ & $\left.\begin{array}{rl}\mathrm{OH} & \rightarrow \mathrm{H} \\
\mathrm{H}^{*} & =5,1 \mathrm{~V} \\
& =10,1 \mathrm{~V}\end{array}\right\}=15,2 \mathrm{~V}$ \\
\hline $\mathrm{H}_{2}$ & $\mathrm{H}_{2} \mathrm{O}^{*} \rightarrow \mathrm{O}+\underbrace{\mathrm{H}+\mathrm{H}}_{\mathrm{H}_{2}{ }^{*}}$ & $\begin{array}{l}\mathrm{OH} \rightarrow \mathrm{H}=\quad 5,1 \mathrm{~V} \\
\mathrm{O} \rightarrow \mathrm{H}=\quad 4,4 \mathrm{~V} \\
\text { Rekombination von } \\
\mathrm{H}+\mathrm{H}=-4,4 \mathrm{~V} \\
\mathrm{H}_{2}{ }^{*} \quad=11,1 \mathrm{~V}\end{array} \mid=16,2 \mathrm{~V}$ \\
\hline $\mathrm{O}$ & $\mathrm{H}_{2} \mathrm{O}^{*} \rightarrow \mathrm{O}^{*}+\mathrm{H}+\mathrm{H}$ & 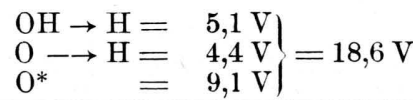 \\
\hline
\end{tabular}

Tab. 1. Verschiedene Zerfallsmöglichkeiten für $\mathrm{H}_{2} \mathrm{O}$ *

Betrachtet man die auftretenden Energien, so erhält man:

Tab. 2. Übersicht über die bei dem Zerfall von $\mathrm{H}_{2} \mathrm{O} *$ auftretenden Energien.

Die Werte für die Abtrennungsenergien sind bei Sponer ${ }^{4}$ entnommen; die Anregungsspannungen sind aus spektroskopischen Daten berechnet.

Die beiden in Tab. 1 am Schluß stehenden Fälle sind in Tab. 2 nicht aufgenommen. Der Fall der Anregung beider Bruchstücke ist noch nicht beobachtet worden, und der Zerfall in zwei unangeregte Teile entzieht sich der Kontrolle, da er durch kein Spektrum sichtbar gemacht wird.

In Abb. 1 ist die energetische Lage der Anregungsniveaus der Bruchstücke mit dem Termschema des Wassers verglichen.

Bei der Erzeugung des $\mathrm{H}_{2} \mathrm{O}$-Spektrums mit der von $\mathrm{Sch}$ üler und Woeldike ${ }^{2}$ entwickelten
Entladungsröhre treten nun die $\mathrm{OH}-\mathrm{Banden}$ deutlich mit den Eigenschaften auf, die für das angeregte Bruchstück aus einem natürlichen Zerfall gefordert werden. Auch die energetischen Verhältnisse liegen für die Anregung bei $\mathrm{OH}$ am günstigsten; aus Tab. 2 ergibt sich, daß der Prozeß $\mathrm{H}_{2} \mathrm{O}^{*} \rightarrow \mathrm{OH}^{*}+\mathrm{H}$ den kleinsten Energieaufwand benötigt. Daß nun der energieärmste Prozeß beobachtet wird, stimmt mit den übrigen experimentellen Befunden überein, die zeigen, daß in der positiven Säule mit ihren geringen Elektronenenergien bevorzugt die kleinsten Anregungszustände beobachtet werden, so daß man das $\mathrm{OH}-$ Spektrum, das bei der Erzeugung von $\mathrm{H}_{2} \mathrm{O}$-Entladungen entsteht, sicher als angeregtes Bruch- 
stück aus dem natürlichen Zerfall $\mathrm{H}_{2} \mathrm{O}^{*} \rightarrow \mathrm{OH}^{*}$ $+\mathrm{H}$ ansehen kann. Es handelt sich um die bekannte $\mathrm{OH}-\mathrm{Bande}$ bei $\lambda=3064 \AA$, die nach Rot abschattiert ist und dem Utbergang $2 \Sigma \rightarrow 2 \Pi$ entspricht.

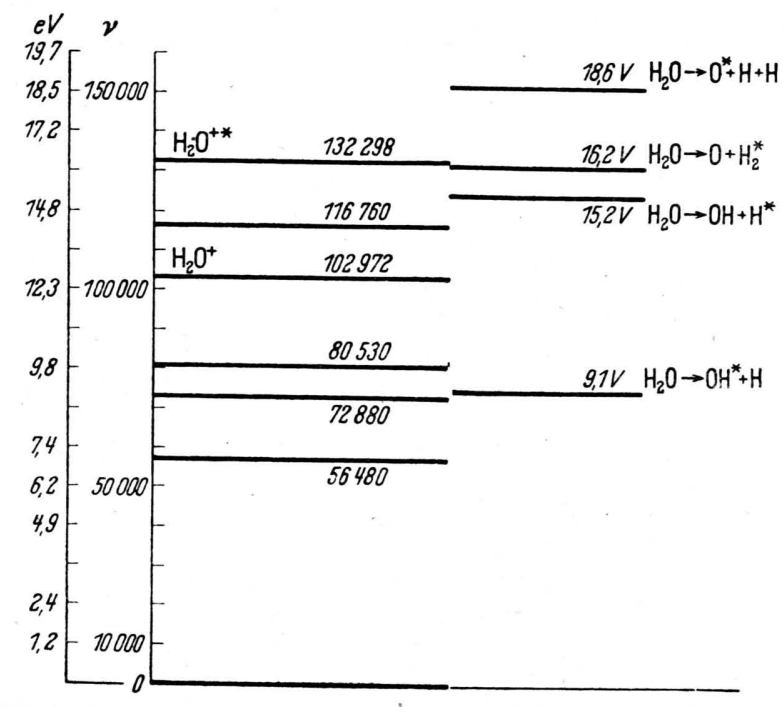

Abb. 1. Termschema des Wassers. Links die Anregungsniveaus der Bruchstücke. scheinlich, da z. B. selbst bei Alkoholen kein $\mathrm{OH}-$ Spektrum gefunden wurde, das dem natürlichen Zerfall zugesprochen werden kann.

Die Experimente wurden mit der Entladungsröhre von $\mathrm{Schüler-Woeldike}{ }^{2}$ durchgeführt, die in ihrer neuesten Form in Abb. 2 dargestellt ist. Die zu untersuchende Substanz gelangt vom Einfüllstutzen $\mathrm{D}$ in den Beobachtungsraum C, dem sich die Kühlfallen $B$ und $B^{\prime}$ anschließen, die in flüssige Luft getaucht werden. Hier werden die Substanzen bis auf das Trägergas ausgefroren, das die Verbindung $\mathrm{zu}$ den Elektroden $\mathrm{A}$ und $\mathrm{A}^{\prime}$ aufrechterhält. $\mathrm{A}$ und $\mathrm{A}^{\prime}$ sind Hohlkathoden, die mit Wasser gekühlt werden. um eine etwaige Erwärmung des Schliff-Fettes zu vermeiden. An beiden Enden wird die Röhre durch aufgekittete Quarzfenster verschlossen. Der Stutzen E dient zum Einfüllen der Substanz.

Bei den Versuchen ist es von ausschlaggebender Bedeutung, daß die Röhre aus Quarz hergestellt wird. Nur dadurch ist es möglich, Verunreinigungen, etwa Wasserhäutchen, schnell und restlos durch Abbrennen mit einem Luft-Leuchtgas-Gebläse zu entfernen. Daß die Röhre durch Kühlen ihrer Kühlfallen mit flüssiger Luft völlig trocken gehalten werden kann, wurde zu Beginn der Versuche durch Kontrollaufnahmen geprüft, die mit reinem $\mathrm{H}_{2}$ gemacht wurden. Bei eingetauchten Kühlfallen waren keine Spuren von $\mathrm{OH}$ zu erkennen, d. h. es befand sich kein $\mathrm{H}_{2} \mathrm{O}$ im Ent-

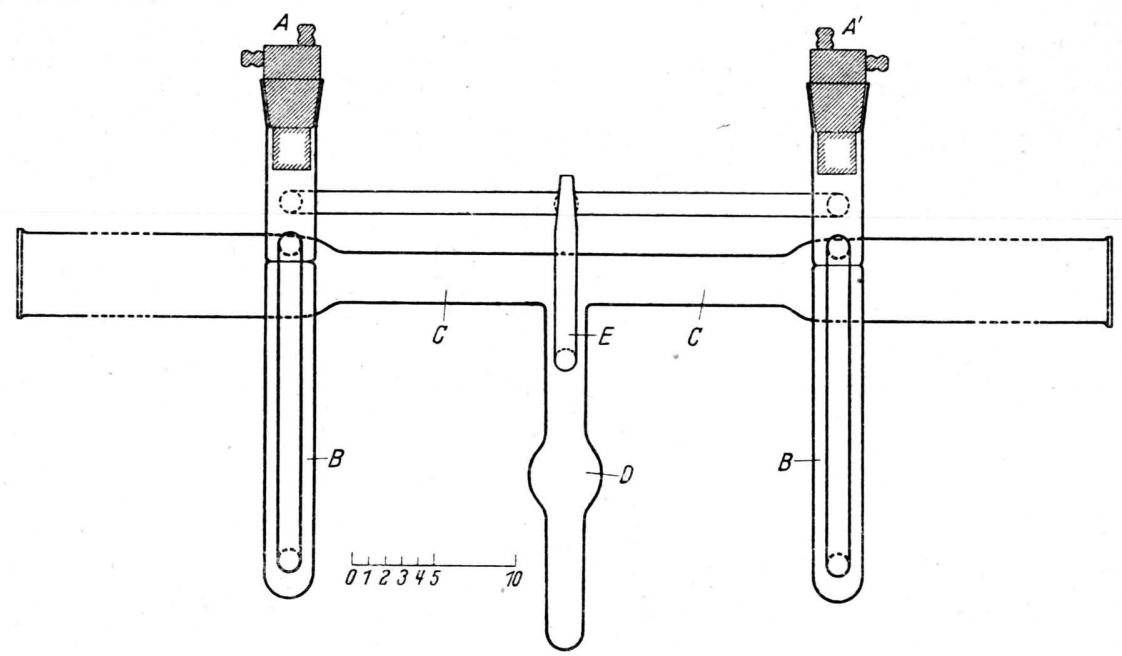

Abb. 2. Die Entladungsröhre von der Seite gesehen.

Findet man also auch bei den geforderten schwachen Stromstärken noch $\mathrm{OH}-\mathrm{Banden}$, so kann man mit Sicherheit auf das Vorhandensein von Wasser schließen. Schwierigkeiten würden dann auftreten, wenn neben $\mathrm{H}_{2} \mathrm{O}$ noch andere Substanzen im Gemisch wären, die OH-Gruppen enthalten, das im natürlichen Zerfall angeregt wird. Es ist dies allerdings nicht sehr wahr- ladungsraum, während bei Fortlassen der Kühlung das OH-Spektrum deutlich sichtbar wurde. Das Kühlen mit flüssiger Luft ist also eine unerläßliche Vorbedingung für quantitative Versuche.

Die Kühlfallen $B$ und $B^{\prime}$ beseitigen zwei weitere Schwierigkeiten, die bisher die Herstellung von Emissionsspektren mehratomiger Moleküle in der Glimmentladung praktisch unmöglich machten. Einmal kommt die zu untersuchende Substanz mit den Elektroden 
nicht in Berührung. So fällt die Verunreinigung durch Zerfall weg, der an den Elektroden so stark ist, daß z. B. organische Substanzen dort bis auf Kohlenstoff abgebaut werden. Der andere Vorteil ist die Möglichkeit, durch das dauernde Ausfrieren der verbrauchten Substanz den Druck etwa konstant zu halten. Man betreibt also die Entladung in der strömenden Substanz.

Um nun festzustellen, welcher Beimengungsgehalt an Wasser spektroskopisch noch nachweisbar ist, wurde das gasförmige Difluor-Dichlormethan als Grundsubstanz gewählt, da es in vorhergehenden Untersuchungen in dieser Apparatur als wasserfrei festgestellt worden war. Beim Zusetzen des $\mathrm{H}_{2} \mathrm{O}$ zur Entladung muß die Röhre geöffnet werden, so daß Luft von Atmosphärendruck in die Apparatur dringt, die nach Kontrollversuchen ein deutliches OH-Spektrum, also Wassergehalt, zeigt. Daher wurde zuerst Difluor-Dichlormethan in die Röhre gefüllt. Es ist schwerer als Luft, verdrängt diese und wird, da der Einfüllstutzen in flüssige Luft getaucht wird, dort verflüssigt und später festgefroren. Der Stutzen ist nunmehr luft- und damit wasserfrei.

In den so vorbereiteten Stutzen wurden nun der Reihe nach verschiedene Wassermengen gebracht. Wassertröpfchen von konstanter Größe (ihr Gewicht war zu $10 \mathrm{mg}$ festgestellt worden) wurden in flüssiger Luft ausgefroren und in die Röhre gebracht. Sie lieferten ein starkes OH-Spektrum. Um kleinere Wassermengen der Entladung zuzuführen, wurden Kapillaren von verschiedener Länge mit Wasser gefüllt und die so definierten Wassermengen in den Einfüllstutzen gebracht. Durch leichtes Erwärmen des Stutzens D (auf etwa $60-70^{\circ} \mathrm{C}$ ) wurde die Feuchtigkeit restlos in die Entladung getrieben. Aufnahmen, die im Anschluß an den eigentlichen Versuch auf die gleiche Platte gemacht wurden, zeigten kein $\mathrm{OH}$-Spektrum mehr.

Die Länge der Kapillaren, und damit die Wassermenge, wurde laufend von $30 \mathrm{~mm}$ bis auf $10 \mathrm{~mm}$ heruntergesetzt. Bei einem Radius von $0,1 \mathrm{~mm}$ bedeutet das eine Wassermenge von $0,9 \mathrm{mg}\left(\mathrm{mm}^{3}\right)$ bis $0,3 \mathrm{mg}$ $\left(\mathrm{mm}^{3}\right)$.

Als vorteilhaftes Trägergas, das die Entladung aufrechterhält, erwies sich im Laufe der Untersuchungen Wasserstoff. Mit ihm wurde die größte Lichtausbeute erzielt. Die Reinheit der Entladung wird durch das Trägergas nicht beeinträchtigt, da es bei genügend großem Dampfdruck der Substanz aus dem Beobachtungsraum verdrängt wird. Der Wasserstoff wird mit Hilfe eines erhitzten Palladiumröhrchens zugeführt.

Der Betrieb der Röhre geht folgendermaßen vor sich: Nachdem die Elektroden in die Schliffe gesetzt sind, wird in den Einfüllstutzen D die Substanz gefüllt und mit flüssiger Luft ausgefroren. Sodann wird die Röhre ausgepumpt; nach Einlassen des $\mathrm{H}_{2}$ ist sie betriebsfertig. Die Verdampfung der Substanz in D wird durch entsprechende Temperaturregelung erreicht.

Die Aufnahmen wurden mit einem Hilger-QuarzSpektrographen auf Agfa-Ultraviolett-Platten gemacht, die zur Erhöhung der Empfindlichkeit vorbelichtet wurden. Die Apparatur wurde nicht mehr mit Gleichstrom, sondern mit Wechselstrom betrieben (der Entladungsweg ist symmetrisch gebaut), der mittels eines Transformators von der üblichen Netzspannung auf $10000 \mathrm{~V}$ bei einer maximalen Stromstärke von $15 \mathrm{~mA}$ transformiert wurde.

Die Entladung wird so geführt, daß in der Beobachtungsrichtung $\mathrm{C}$ nur die positive Säule liegt. Hier liegen die Potentialverhältnisse so, daß die Elektronengeschwindigkeiten im allgemeinen nicht über die Ionisierungsspannungen des jeweiligen Gases hinauskommen. Der größte Teil der zu untersuchenden Moleküle aber erfährt seine Anregung von Elektronen mit langsameren Geschwindigkeiten. Daher zeichnen sich bei Spektren der positiven Säule die ersten Anregungszustände der Atome und Moleküle durch besonders starke Intensität aus.

Kleinere Wassermengen als $0,3 \mathrm{mg}$ waren mit dieser Apparatur nicht mehr nachweisbar. Da der Einfüllstutzen $60 \mathrm{~g}$ Difluor-dichlormethan faßte, war das Mischungsverhältnis 1:180000. Dieses ist durch die Dimensionierung der Röhre bedingt. Bei entsprechender Vergrößerung des Behälters muß sich ein noch günstigeres Verhältnis erzielen lassen, denn es ist prinzipiell für den Nachweis des Wassers gleichgültig, ob es sich allein oder mit einer gewissen Menge Fremdsubstanz in der Apparatur befindet, solange deren Spektrum das der $\mathrm{OH}$-Gruppe nicht überdeckt.

Man kommt mit diesem Nachweis von Wasser als Beimengung im Verhältnis 1:180000 in den Empfindlichkeitsbereich der Metallanalysen. Unter konstanten Versuchsbedingungen und bei Verwendung von Testproben ist es sicher möglich, auch quantitative Aussagen über den Wassergehalt von Gemischen zu machen.

Hrn. Prof. S c h ü l e r möchte ich für das fördernde Interesse, das er dieser Arbeit entgegenbrachte, sowie für die Möglichkeit der Beteiligung an seinen Untersuchungen herzlich danken. 